\title{
Multiresistente Keime
}

\section{Thomas Mückley, Michael Diefenbeck, Gunther Olaf Hofmann}

\section{Zusammenfassung}

Die aktuelle Prävalenz Methicillin-resistenter Staphylococcus - aureusStämme (MRSA) von 20,7\% in Deutschland, eine mögliche Ausbreitung von cMRSA und die erschwerte effektive Therapie von manifesten MRSA-Infektionen kann bei knapper werdenden finanziellen und personellen Ressourcen im Gesundheitssystem schnell zu kritischen Situationen führen. Der strikten Einhaltung krankenhaushygienischen Maßnahmen, der effektiven Infektionskontrolle, der Im-

\section{Einleitung}

Die seit Jahren steigende Prävalenz der multiresistenten Erreger, im Speziellen von Methicillin-resistenten Staphylococcus aureus-Stämmen (MRSA), ist alarmierend und kann in Kombination mit einer erschwerten effektiven Antibiotikatherapie bei knapper werdenden finanziellen und personellen Ressourcen im Gesundheitssystem schnell zu kritischen Situationen führen. Die zunehmende Konzentrierung schwer- und schwerstkranker Patienten in den stationären Versorgungsbereichen, die steigende Zahl invasiver medizinischer Maßnahmen sowie der häufige unkontrollierte Einsatz von Breitbandantibiotika tragen zur Selektion und Verbreitung mulitresistenter Erreger bei [22].
OP-JOURNAL 2005; 21: 260-268

(c) Georg Thieme Verlag KG Stuttgart · New York plementierung von Präventionsstrategien und der adäquaten antimikrobiellen Therapie kommt in der Senkung der MRSA-Prävalenz eine zentrale Stellung zu. MRSA-Infektionen des muskuloskelettalen Systems bedürfen neben allgemeinen Hygienemaßnahmen ein konsequentes chirurgisches und antibiotisches Vorgehen. Häufig sind auf dem Weg zur Infektsanierung komplexe Revisionseingriffe erforderlich. Infektrezidive bei diesen Patienten müssen bis zum Beweis des Gegenteils als MRSA-positiv eingestuft werden.

\section{Hauptteil}

\section{Definition}

Die Abkürzung MRE steht ganz allgemein für multiresistente Erreger (Abb.1). Man versteht darunter Krankheitserreger, die aufgrund ihrer Resistenzen nur noch sehr eingeschränkt oder überhaupt nicht mehr antibiotisch behandelt werden können. Betroffen davon sind keinesfalls nur die Staphylokokken sondern zunehmend auch andere Erreger (z.B. Enterokokken, Pseudomonaden).

- MRSA = Methicillin-resistenter Staphylococcus aureus

- $\mathbf{O R S A}=$ Oxacillin-resistenter Staphylococcus aureus

- MRSE = Methicillin-resistenter Staphylococcus epidermidis

- ORSE = Oxacillin-resistenter Staphylococcus epidermidis

MRSA und ORSA bzw. MRSE und ORSE werden im klinischen Alltag synonym verwendet.

MRSA-Stämme weisen gegenüber allen Betalaktamantibiotika eine kreuzresistenz auf.

MRSA-Stämme zeichnen sich dadurch aus, dass sie gegenüber allen Betalaktamantibiotika (Penicilline, Cephalosporine, Carbapeneme) eine Kreuzresistenz aufweisen. Außerdem besitzen viele Stämme eine zusätzliche Unempfindlichkeit gegenüber anderen Antibiotika-Substanzklassen und werden deshalb als multiresistente Erreger bezeichnet [36]. Gleichzeitig weisen sie Mehrfachresistenzen gegen Chinolone, Aminoglykoside, Makrolide und Lincosamide auf.

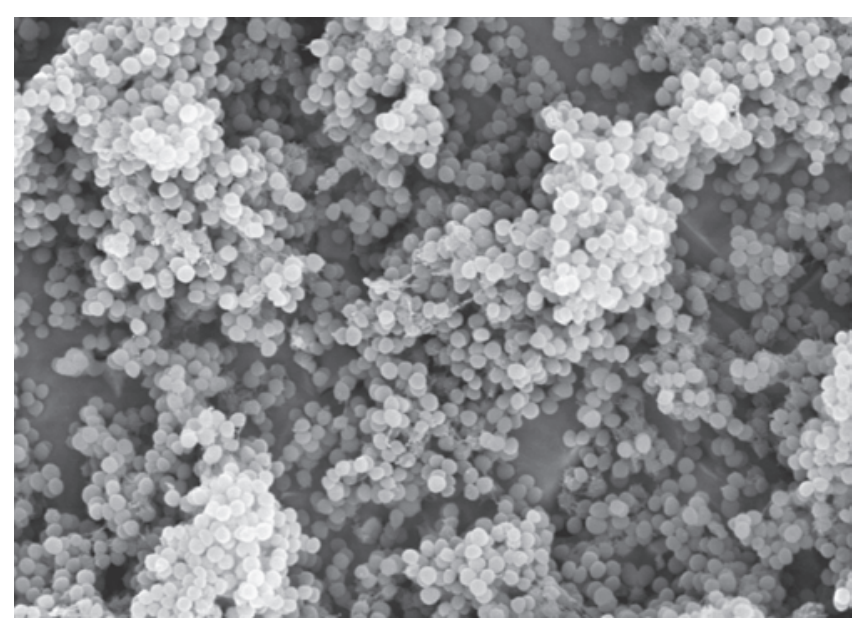

Abb. 1 Mikroskopisches Bild einer Staphylokokkenkolonie (MRSA) auf einer Implantatoberfläche. 
Tab. 1 Erfassungspflichtige Erreger und ihre Resistenzen nach §23(1) des Infektionsschutzgesetzes

Erregerspezies

Zu erfassen ist die Resistenz (auch Einzelresistenzen) gegen folgende Substanzen, sofern im Rahmen der klinisch mikrobiologischen Diagnostik getestet

1 Staphylococcus aureus

2 Streptococcus pneumoniae

3 Enterococcus faecalis Enterococcus faecium

4 Escherichia coli, Impidenem/Meropenem, Chinolon Gr II (z. B. Ciprofloxacin), Klebsiela spp. Amikacin, Ceftazidim, Piperacillin/Tazobactam, Cefotaxim

5 Enterobacter cloa- Imipenem/Meropenem, Chinolon Gr. II (z. B. Ciprofloxacin), cae, Citrobacter spp. Serratia marcescens

6 Pseudomonas ae- Imipenem/Meropenem, Chinolon Gr. II (z. B. Ciprofloxain), ruginosa, Acineto- Amikacin, Ceftazidim, Piperacillin/Tazobactam bacter baumannii

7 Stenotrophomonas maltophilia

8 Candida spp.

Chinolon Gr. II (z. B. Ciprofloxacin), Amikacin, Cefazidim, Piperacillin/ Tazobactam

Fluconazol
Der Begriff der „Mehrfach oder „Multiresistenz" von Keimen ist auch juristisch definiert: Das Infektionsschutzgesetz (IfSG) [19] definiert jene Erreger mit Mehrfachresistenzen, die erfasst werden müssen (Tab.1).

Nach $\S 23$ Abs. 1 des Infekionsschutzgesetz besteht seit dem 1.1.2001 für Krankenhäuser und Einrichtungen für ambulantes Operieren die Verpflichtung zur Erfassung und Bewertung von Erregern mit Resistenzen und Multiresistenzen sowie von bestimmten nosokomialen Infektionen [17]. Diese Daten ergänzen die teilweise schon in Krankenhäusern verfügbaren lokalen Keimhäufigkeits- und Resistenzstatistiken. In $§ 4(2) 2$ des IfSG sind die Keime dazu benannt. Bei MRSA-Epidemien müssen die Stämme aufgehoben und typisiert werden [7]. Die Aufzeichnungen sind zehn Jahre aufzubewahren. Dem zuständigen Gesundheitsamt ist auf Verlangen Einsicht in die Aufzeichnungen $\mathrm{zu}$ gewähren. Bei vermehrtem Auftreten eines klonal identischen MREStammes in räumlich und zeitlichem $\mathrm{Zu}$ sammenhang ist das zuständige Gesundheitsamt zu informieren ( $\$ 6$ IfSG).

\section{Häufigkeit und Verbreitung}

Staphylococcus aureus ist ein ubiquitär vorkomender Erreger, der bei 15-40\% der Menschen zur normalen Haut- und Schleimhautflora gehört. Durch eine hohe Umweltresistenz (Persistenz) kön- nen diese Erreger über Monate in der unbelebten Umwelt überleben und nachfolgend über kontaminierte Hände oder Gegenstände auf andere Personen übertragen werden. Etwa 30\% aller stationären Patienten werden innerhalb der ersten 5-10 Tage eines Krankenhausaufenthaltes mit Staphylococcus aureus besiedelt.

Ursprünglich waren die verschiedenen Staphylokokkenspezies gegenüber Antibiotika nahezu uneingeschränkt empfindlich. Im Laufe der 50er-Jahre konnte

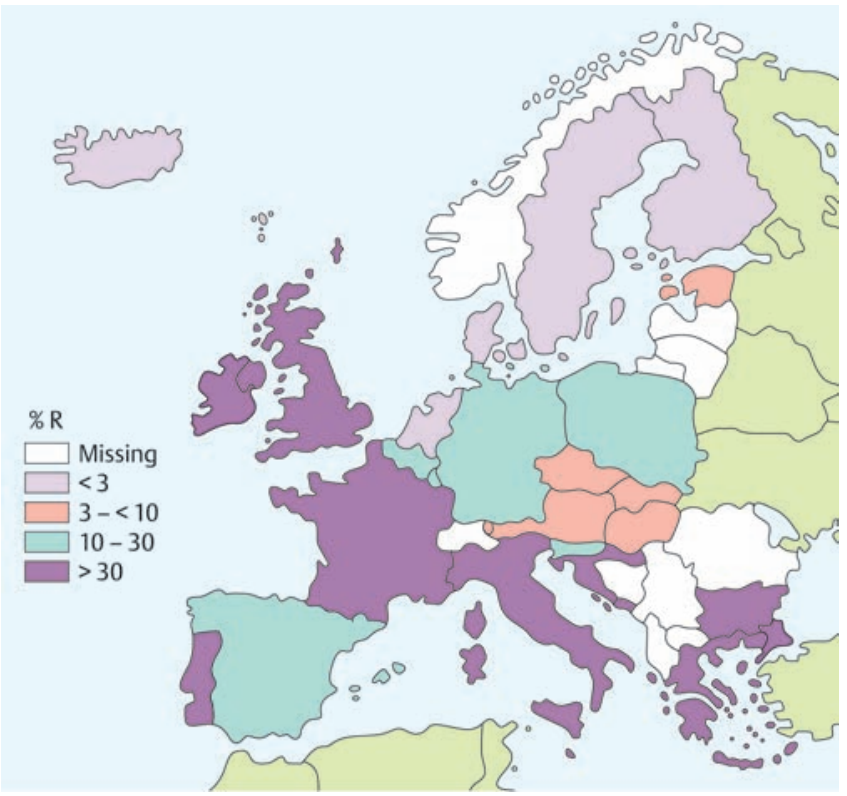

Penicillin wegen des Auftretens Penicillinase-bildender Staph.-aureus-Stämme nicht mehr umfassend eingesetzt werden. Anfang der 60er-Jahre wurden Methicillin und später weitere Isoxazolylpenicilline (Oxacillin, Flucloxacillin) als so genannte penicillinasefeste Penicilline eingeführt. Aber bereits in den 60er-Jahren wurde in England der erste Methicillin-resistente Staphylococcus aureus (MRSA) nachgewiesen [37].

In den Jahren 1996 und 1997 wurde in Japan und den USA erstmals über MRSAStämme mit einer verminderten Empfindlichkeit gegenüber Glykopeptidantibiotika berichtet [32]. Besorgniserregend ist die vor kurzem erstmalige Isolierung eines Staph. aureus mit kompletter Resistenz gegen Vancomycin in den USA [12].

In Europa gibt es bezüglich der MRSARate ein deutliches Nord-Süd-Gefälle (Abb. 2). In Norwegen, Schweden, Dänemark und den Niederlanden liegt die MRSA-Rate unter $1 \%$, wohingegen sie in Portugal, Spanien und Griechenland über 30\% liegt [7,33]. In Deutschland konnte zwischen 1975 und 1995 ein stetiger Anstieg des Anteils Oxacillin-resistenter Staphylokokken festgestellt werden [24]. Im Zeitraum von 1990 und 1998 hat sich in deutschen Krankenhäusern die Prävalenz von MRSA nahezu verzehnfacht (1990: 1,7\%; 1998: 15,2\%) [42]. Mittlerweile liegt ihr Anteil in der BRD im Jahr 2001 bei 20,7\%. In Japan und den USA liegen die Resistenzquoten sogar zwischen 40 und 50\% [1].
Abb. 2 Anteil von MRSA an Staphylococcus aureus in Blutkulturen in Europa von 19992001 (EARSS - European Antimicrobial Resistance Surveillance Study Annual Report 2001). 
Es gibt zahlreiche prädisponierende Faktoren für eine Besiedlung mit MRSA und anderen multiresistenten Keimen: hierzu zählen u.a. Diabetes mellitus, Niereninsuffizienz, Psoriasis, Fremdkörperimplantate (Katheter, Sonden, Endoprothesen), Verbrennungen, große Wunden und Resistenzminderung durch chronische Erkrankungen.

\section{Bedeutung}

Staphylococcus aureus gilt v. a. als Erreger von abszedierenden Infektionen im Bereich der Haut und Weichteile. Er spielt jedoch auch bei postoperativen Wundinfektionen, chronischen Koncheninfektionen, Gelenkinfektionen und Protheseninfektionen eine wesentliche Rolle.

Die zunehmende Konzentrierung schwer- und schwerstkranker Patienten in den stationären Versorgungsbereichen, die steigende Zahl invasiver medizinischer Maßnahmen sowie der häufige unkontrollierte Einsatz von Breitbandantibiotika tragen zur Selektion und Verbreitung multiresistenter Erreger bei [22]. Weltweit hat in den letzten Jahren die Anzahl der Stämme, die Methicillinresistent sind, sprunghaft zugenommen.

Wegen ihrer eingeschränkten Therapierbarkeit stellen Infektionen durch MRSA für den betroffenen Patienten, aber auch für das Krankenhaus ein besonderes medizinisches und ökonomisches Problem dar.

Die MRSA-Kolonisation ist dabei von der MRSA-Infektion zu unterscheiden. Aus einer vorbestehenden MRSA-Kolonisation kann sich jederzeit eine manifeste MRSA-Infektion entwickeln.

Auswirkungen auf die muskuloskeletale Chirurgie

Die MRSA-Problematik betrifft insbesondere die chirurgischen Kliniken. In der Mehrzahl der europäischen Länder wird MRSA zu einem relevanten Prozentanteil bei Untersuchungsmaterialien von chirurgischen Patienten isoliert [33].

Bei vielen Eingriffen in der muskuloskelettalen Chirurgie werden Implantate bzw. Prothesen eingebracht. Die in vielen Krankenhäusern standardisiert eingesetzte Antibiotikaprophylaxe ist gegen die meisten multiresistenten Keime unwirksam. Ebenso sind bei eingetretener Infektion mit multiresistenten Erregern die gängigen lokalen Antibiotikaträger

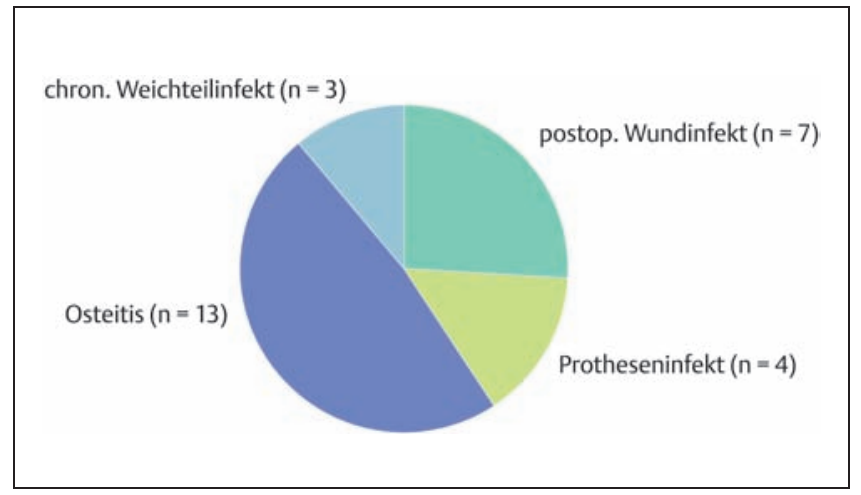

Abb. 3 Prozentuale Verteilung der MRSA-Infektionen bei Erkrankungen in der septischen Extremitätenchirurgie (eigene Daten).

in der Regel nicht wirksam. Eine effektive systemische Antibiotikatherapie in diesen Fällen gelingt nur mit dem Einsatz von Reserveantibiotika. Hierbei wird der Neueinführung von Antibiotika viel Hoffnung entgegengebracht.

Betrachtet man die Einführung von Linezolid in den Jahren 2000/2001, so zeigte dieses erste Oxazolidinon mit neuem Wirkmechanismus keine Kreuzresistenz zu anderen Antibiotikaklassen. Das deutlich kleinere Molekulargewicht im Vergleich zu Vancomycin erleichterte auch die Gewebepenetration, die gerade bei Infektionen von muskuloskelettalem Gewebe von Bedeutung ist. Die erste Resistenz von MRSA auf Linezolid wurde bereits 2001 nachgewiesen und verdeutlicht, dass die Kolonisation bzw. Infektion mit multiresistenten Erregern nicht langfristig durch die Neuentwicklung von potenten Antibiotika eingedämmt werden kann [40].

Auch die klinischen Ergebnisse bei Infektionen mit MRSA, speziell nach Operationen mit Implantationen von alloplastischen Materialien, stimmen nachdenklich. Publikationen aus der Gefäßchirurgie geben Mortalitätsraten von 56-100\% nach aortoiliakalen Rekonstruktionen bzw. alloplastischem Aortenersatz mit nachfolgender MRSA-Infektion an [14,31]. Kilgus et al. fanden für die Hüft- und Knieprotheseninfektionen mit MRSA deutlich schlechtere Ergebnisse im Vergleich zu Methicillin-sensitiven Staphylococcus-aureus-Protheseninfektionen [21].

Die Ergebnisse der Therapie von MRSAProtheseninfektionen sind schlechter als die bei Protheseninfektionen mit „Normalkeimen“.

Die Probleme im Rahmen der Behandlung von Infektionen am muskuloskelettalem System konnten wir am eigenen Patien- tengut nachvollziehen [30]. In einer prospektiven Studie zwischen Januar 2000 bis Januar 2002 schlossen wir 27 Patienten (20 Männer, 7 Frauen) mit einem Durchschnittsalter von 54 Jahren (15 bis 75 Jahre) mit nachgewiesenen MRSA-Infektionen ( 7 postoperative Wundinfektionen, 4 Protheseninfektionen, 13 chronische Knochen- und 3 chronische Weichteilinfektionen) ein (Abb.3). Besiedlungen mit MRSA wurde nicht berücksichtigt. Bei 8 von 24 zuverlegten Patienten war der MRSA-Status zum Verlegungszeitpunkt nicht bekannt oder wurde nicht entsprechend übermittelt. Die Vorbehandlung erstreckte sich im Mittel über 9,7 Monate. Bei 74,1\% der Patienten bestanden relevante Vorerkrankungen bzw. Risikofaktoren für eine MRSA-Infektion. Das therapeutische Konzept umfasste radikale Etappen-Debridements mit mikrobiologischen Probeentnahmen, Vakuumverbänden und die testgerechte lokale und systemische Antibiotikatherapie. Nach 3 negativen Wundabstrichen erfolgte der Wundverschluss und die Aufhebung der Isolation. Der stationäre Aufenthalt belief sich auf durchschnittlich 66 Tage (17 bis 187 Tage) mit einer durchschnittlichen OP-Anzahl von 7,3 Operationen. Die Patienten mussten durchschnittlich 23 Tage in Isolation verbringen. Während des Behandlungszeitraumes wurde keine Übertragung von MRSA auf andere Patienten nachgewiesen. Alle MRSA-Infektionen konnten bis zum Rückgang der Infektionszeichen und bis zum Vorliegen von negativen Wundabstrichen behandelt werden. Es mussten jedoch bei 13 der 27 behandelten Patienten folgenschwere Eingriffe (Lappenplastiken, Segmentresektionen, Arthrodesen, Amputationen, Girdelstone-Situation) durchgeführt werden (Abb.4). Zum Zeitpunkt der Nachuntersuchung nach durchschnittlich 22 Monaten zeigten sich 4 Infektrezidive (14,8\%). Alle Infektrezidive waren erneut MRSA-positiv. 


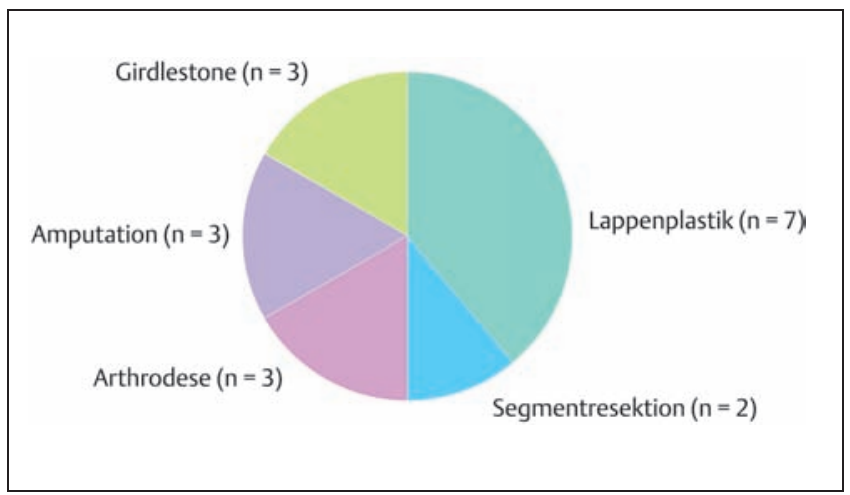

Abb. 4 Auflistung der durchgeführten folgenschweren operativen Eingriffe.
Somit waren bei fast der Hälfte der Patienten mit MRSA-Infektionen des muskuloskelettalen Systems komplexe Revisionseingriffe erforderlich. Da die aufgetretenen Infektrezidive allesamt MRSApositiv waren, wurde von uns die Empfehlung ausgesprochen, alle Patienten mit Rezidiven nach vorangegangenen MRSA-Infektionen primär, am Aufnahmetag auf MRSA zu screenen.

Die Probleme in der Therapie von manifesten MRSA-Infektionen des muskuloskelettalen Systems verdeutlichen, dass zukünftig der Prävention von MRSAÜbertragungen eine besondere Bedeutung zukommt. Hierbei ist zu beachten, dass die schlechten Ergebnisse der Therapie am ehesten auf die bestehenden Grunderkrankungen der Patienten und die eingeschränkten Therapiemöglichkeiten zurückzuführen sind. Entgegen früheren Auffassungen ist MRSA in Bezug auf invasive Infektionen nicht weniger oder mehr virulent als Methicillin-sensitiver Staphylococcus aureus.

\section{Auswirkung auf den ambulanten Bereich}

Aktuelle bevölkerungsbasierte Studien der CDC (Center of Disease Control and Prevention) bestätigen, dass MRSA nicht lediglich ein Problem der Krankenhäuser und der entsprechenden Pflegeeinrichtungen ist. MRSA etabliert sich in den USA mittlerweile auch außerhalb von Kliniken. Die Rate der „community acquired“ MRSA (cMRSA=MRSA-Nachweis bei Patienten ohne Kontakt zu medizinischen Einrichtungen oder Risikofaktoren) liegt in den USA schon bei 8-20\% [10]. In Deutschland waren im Jahr 2004 1,1\% der Staphylococcus-aureus-Isolate außerhalb von Kliniken Methicillin-resistent. Aktuelle Daten zum Vorkommen in Ostbayern zeigen jedoch bereits eine weite Verbreitung dieser Keime außerhalb von Krankenhäusern mit erheblichen Konsequen- zen für Diagnostik, Hygienemaßnahmen und Therapie [8]. Dies bedeutet, dass nun auch zunehmend niedergelassene Ärzte mit MRSA-Fällen konfrontiert werden.

MRSA etabliert sich mittlerweile auch außerhalb der Krankenhäuser.

Im Unterschied zu herkömmlichen Staphylococcus-aureus-Stämmen besitzen cMRSA-Stämme eine einzigartige Kombination von Pathogenitäts- und Resistenzfaktoren. Hervorzuheben ist der Besitz des Panton-Valentine-Leukozidins (PVL), das klinisch zum Auftreten von rezidivierenden tiefen Haut- und Weichteilinfektionen führen kann, typischerweise oft ohne klassische Eiterbildung oder erkennbare Eintrittspforte. In seltenen Fällen kommt es zu nekrotisierenden Pneumonien mit hoher Letalität, die entsprechend ihres akuten Verlaufs häufig in der Tagespresse Niederschlag finden.

\section{Resistenzmechanismus}

Der Methicillin/Oxacillin-Resistenz liegt der Einbau einer zusätzlichen DNA-Sequenz in das Chromosom zugrunde (mecA-Gen). Dadurch wird ein neues Penicillinbindeprotein (PBP 2a) kodiert mit geringer Affinität zu Methicillin/Oxacillin und allen Betalaktamantibiotika. Zum Ursprung von MRSA gibt es die vorherrschende Theorie, dass das mecA-Gen vor Jahrzehnten von relativ wenigen Staph.-aureus-Stämmen horizontal via Transposition erworben wurde [23]. In der Folgezeit ist es zur Ausbreitung der Stämme gekommen, wofür zum einen der Selektionsdruck von Breitspektrumantibiotika und zum anderen hygienische Mängel verantwortlich sind.
Identifikation von mehrfachresistenten Keimen

Material

Die mikrobiologische Diagnostik stellt eine wesentliche Säule bei der Behandlung von Patienten mit Knochen- und Knorpelinfektionen dar; ein Erregernachweis ist als Grundlage eines rationalen Therapiekonzeptes unbedingt erforderlich. Das Untersuchungsmaterial wird im Regelfall invasiv gewonnen - am besten geeignet sind Biopsien und Punktate [15] - und sollte innerhalb von zwei Stunden im Labor verarbeitet werden. Empfehlenswert ist die Verwendung von Flüssig- und Festnährmedien und die Bebrütung über 10-14 Tage.

\section{Bestimmung der Methicillinresistenz}

Die übliche Resistenzbestimmung erfolgt mittels Agardiffusionstest zur Bestimmung des Hemmhofdurchmessers nach deutschen oder amerikanischen Normen (DIN bzw. NCCLS). Diese Methode nimmt mindestens zwölf Stunden in Anspruch und kann bei bestimmten Resistenzphänomenen, wie heteroresistenten MRSAStämmen, an ihre Grenzen geraten und spezielle zusätzliche Techniken, wie ETests, erforderlich machen.

Die MHK-Bestimmung mittels Bouillondilutionstest stellt die Standardmethode für die Bestimmung der Empfindlichkeit gegen Oxacillin dar, ist aber als Routineverfahren zu umständlich. Automatisierte Identifizierungs- und Resistenzstimmungssysteme wie Vitek2 ${ }^{\circledR}$, Phoenix $^{\circledR}$ und MicroScan Walkaway ${ }^{\circledR}$ ersetzen zunehmend die klassischen manuellen Verfahren. Dabei wird das Bakterienwachstum in Anwesenheit von mindestens drei Konzentrationen eines Antibiotikums im Vergleich zur Wachstumskontrolle gemessen und die Rohdaten mithilfe eines spezifischen Algorithmus in die MHK umgerechnet.

Eine neue attraktive Methode zur Detektion der Oxacillin-Resistenz ist der Nachweis des PBP2a mittels Latexagglutination mit einem für $\mathrm{PBP} 2 \mathrm{a}$ spezifischen monoklonalen Antikörper.

Referenzmethode für den Nachweis der Oxacillin-Resistenz ist die molekulargenetische Analyse des mecA-Gens mittels PCR. 


\section{Typisierungsverfahren}

Seit 1992 wird in Deutschland eine überregionale Ausbreitung bestimmter MRSA-Klone in den Krankenhäusern beobachtet; im Wesentlichen sind dies - benannt nach der Region ihres erstmaligen Auftretens - der Norddeutsche, der Süddeutsche, der Hannoversche, der Wiener, der Berliner, der Barnim- und in den letzten Jahren mit einer sehr schnellen Ausbreitungstendenz der Rhein-Hessen-Epidemiestamm [43].

Da MRSA-Stämme die Tendenz haben, Ausbruchsituationen zu verursachen, liefert die Erregertypisierung einen wesentlichen Beitrag, um epidemische von sporadisch auftretenden Stämmen zu differenzieren und Infektketten sowie Streuquellen aufzudecken [41]. Man unterscheidet phänotypische und genotypische Methoden:

- Die Phänotypisierung charakterisiert den Erreger nach seinem Erscheinungsbild wie dem Antibiotikaresistenzprofil.

- Genotypische Verfahren beruhen auf der Analyse genetischer Strukturen, welche unabhängig von äußeren Einflüssen sind, und können über DNSPolymorphismen die Erreger differenzieren.

Es existieren verschiedene molekulargenetische Methoden: Plasmidanalyse, Restriktionsenzymanalyse von DNA, Restriktions - Fragment - Längenpolymorphismus (RFLP), AP-PCR (Arbitrarily Primed Polymerase Chain Reaction), PCR-DNA, sequenzbasierte Typisierung und PFGE (Pulsfeldgelelektrophorese), wobei letztgenannte nach wie vor als Goldstandard gilt [39].

\section{MRSA-Management}

Die steigende Prävalenz von MRSA und die zunehmende Anzahl von betroffenen Krankenhäusern sind u.a. auf Fehler im Hygieneregime und nicht ausreichende Informationsübermittlung an die Nachfolgeeinrichtungen bei Verlegungen von MRSA-Patienten zurückzuführen [42]. Auch im klinischen Alltag zeigen sich mitunter Tendenzen, MRSA-positive Patienten rasch $\mathrm{zu}$ verlegen und empfohlene Hygienemaßnahmen zu umgehen [13]. Vorrangig ist sicherlich die Übertragung durch die Hände des medizinischen Personals. Um eine weitere Ausbreitung von MRSA in Deutschland zu verhindern, ist ein striktes Hygienemanagement erforderlich $[7,17,42]$.
Vorrangig ist die Übertragung von MRSA durch die Hände des medizinischen Personals.

Ein effektives MRSA-Management setzt sich aus verschiedenen Komponenten zusammen:

- primäres Screening

- krankenhaushygienische Maßnahmen

- „kontrollierter“ Antibiotikaeinsatz

- Kommunikation und Information

- Fort- und Weiterbildung.

Kosten-Nutzen-Analysen haben ergeben, dass Screeningprogramme zur Identifizierung unerkannter MRSA-Träger bei der Krankenhausaufnahme effektiv sind. Sie tragen zur Senkung der MRSA-Übertragung bei und vermindern dadurch die erheblichen Kosten für zusätzliche Isolierungsmaßnahmen. Die wichtigsten Hygienemaßnahmen bei MRSA-Patienten sind bei Fachgesellschaften wie der AWMF (www.awmf-online.de) oder Instituten wie dem RKI (www.rki.de) detailliert abrufbar und umfassen u.a.:

- räumliche Isolierung

- mikrobiologisches Eingangsscreening

- erheben des Kolonisationsstatus

- strikte Händehygiene

- Anlegen von Einmalhandschuhen, Schutzkitteln und Mundschutz bei Patientenkontakt

- Desinfektion der patientennahen Flächen, patientenbezogenen Gebrauchsgegenstände

- Indikationsstellung einer Eradikationstherapie

- Dokumentation aller durchgeführten Maßnahmen und entsprechende Information und Aufklärung von Patienten, Personal und Besuchern.

Neben diesen Maßnahmen sind auch baulich-funktionelle und organisatorische Faktoren sowie die Personaldichte bei einem effektiven MRSA-Management mit zu berücksichtigen.

\section{Umgang mit MRSA-Patienten}

Es besteht Konsens darüber, dass dem Risiko einer Verbreitung von MRSA mit genau definierten Hygienemaßnahmen begegnet werden muss. Der Hauptübertragungsweg von MRSA erfolgt über Kontakt mit den Händen des Patienten sowie des Krankenhauspersonals. Die wichtigste und effizienteste Maßnahme, diesen Übertragungsweg zu unterbrechen, ist die konsequente und sachgerechte Händedesinfektion sowie das Tragen von Schutzhandschuhen.

\section{Maßnahmen bei MRSA-Verdachtsfall}

Das Auftreten eines MRSA-Verdachtsfalles sollte sogleich zu folgenden Maßnahmen führen:
- Wiederholung des Wundabstriches
Nasenabstrich
- Rachenabstrich
- Perinealabstrich
Inguinalabstrich.

Bei den Mitpatienten im gleichen Zimmer sollten zeitgleich zumindest erfolgen:

- Nasenabstrich

- Rachenabstrich

ggf. Wundabstrich.

Das Auftreten eines MRSA-Falles erfordert die unverzügliche Informationsweitergabe an den mit der Hygiene befassten Personenkreis (z.B. Hygienebeauftragter). Außerdem sollte eine Rückmeldung des Erkrankungsfalles an die vorbehandelnden/vorbetreuenden Personen und Institutionen erfolgen, damit auch dort die notwendigen Maßnahmen ergriffen werden können.

\section{Maßnahmen bei MRSA-Nachweis}

Bei MRSA-Nachweis wird die Isolation des Patienten möglichst im Einzelzimmer mit eigener Nasszelle oder die Kohortenisolation mehrerer MRSA-Patienten (mit identischen MRE-Stämmen) empfohlen.

Patienten mit MRSA-Nachweis sind im Krankenhaus räumlich zu isolieren.

Für die weitere Betreuung des Patienten sollte die Anzahl der erforderlichen Personen minimal gehalten werden. Dies betrifft auch ärztliche Visiten und Verbandswechsel.

Bei Pflegemaßnahmen bzw. Kontakt mit dem Patienten sind besondere Schutzmaßnahmen, wie Kittelpflege, das Tragen von Schutzhandschuhen, einer Kopfhaube und eines Mundschutzes zur Vermeidung einer Keimakuisition und Übertragung empfehlenswert. Prinzipiell sollten Besuche dieser Patienten auf ein vertretbares Minimum reduziert werden. Alle Besucher sind auf die notwendigen Hygienemaßnahmen hinzuweisen. Vor dem Betreten des Zimmers sind Kittel, Mund- und Nasenschutz, Kopfhaube und Einmalhandschuhe anzulegen. Nach dem Verlassen des Zimmers gibt es klare Vorgaben für die Entsorgung der Schutzkleidung (AWMF-Leitlinie Nr. 029/019). Die anschließende Händedesinfektion ist obligat. 
Der Patient darf das Isolierzimmer nicht verlassen (Ausnahme: eigener Balkon, eigene Terrasse).

Bei Patienten mit MRSA-Infektionen sollten Transportmaßnahmen im Krankenhaus (auf Transportliege, nicht im Bett, mit Mund-/Nasenschutz) nur bei strenger medizinischer Indikation durchgeführt werden. Transportmaßnahmen und operative Maßnahmen sind als MRSA-positiv anzukündigen und zu planen. Ob MRSAEingriffe in speziellen septischen Operationssälen und am Ende des regulären Operationsprogramms erfolgen müssen, wird zwar kontrovers diskutiert [5,7], sollte unseres Erachtens aber so gehandhabt werden. Vom Robert-Koch-Institut werden Operationen an MRSA-positiven Patienten als „septisch“ eingestuft. Vor der Operation sollten alle beweglichen und entbehrlichen Gegenstände aus dem Saal entfernt werden. Der Saal ist während des Eingriffes und bis zum Abschluss aller Desinfektionsmaßnahmen streng zu isolieren. Ein MRSA-Patient darf nicht in den Aufwachraum, die Narkose ist im Operationssaal auszuleiten und die Weiterüberwachung muss auf Station erfolgen.

\section{MRSA-Eradikation}

Die MRSA-Kolonisation ist von großer Bedeutung für die Weiterverbreitung von MRSA. Am häufigsten betroffene Bereiche sind der Nasopharynx und die Haut.

Eine Eradikation von MRSA aus der Nase ist durch lokale Anwendung von Antibiotika (z.B. Mupirocin-Nasensalbe, $3 \times$ täglich über 5 Tage) möglich. Die Mund-Rachen-Antiseptik muss mit einem anerkannt wirksamen mikrobioziden Wirkstoff durchgeführt werden.

Eine Kolonisation der Haut ist wesentlich schwerer zu behandeln. Es liegen bisher keine Daten über effektive Methoden der Behandlung einer MRSA-Kolonisation der Haut vor. Empfohlen wird ein antiseptisches tägliches Ganzkörperbad (Polihexamid, Octenidin, Chlorhexidinseife) unter Einbeziehung der Kopfhaare (je 15 Minuten über 3-7 Tage). Frühestens drei Tage nach Abschluss der Sanierungsmaßnahmen müssen Kontrollabstriche an drei aufeinander folgenden Tagen durchgeführt werden.

\section{Probleme}

Die Umsetzung der Hygienevorgaben erfordert Bettenkapazitäten (Isolierstation) und einen hohen Stellenschlüssel im Pflegebereich (Intensivstandard). Merkblätter und Dokumentationsbögen erleichtern es, die notwendigen Maßnahmen durchzuführen und liegen mittlerweile in den meisten betroffenen Krankenhäusern vor. Regelmäßige Information über diese Problematik ist zwingend.

Aufgrund der häufigen Thematisierung von MRSA in der nicht medizinischen Presse ist für die Zukunft aber mit deutlich aufgeklärteren, aber auch stärker verunsicherten Patienten bezüglich MRSA zu rechnen. Hieraus ergeben sich mögliche zukünftige Spannungsfelder. Bei erstmaligen MRSA-Nachweisen sowie bei MRSA-Übertragungen im Rahmen von stationären Aufenthalten könnte die Einhaltung und Effizienz der Präventionsund Hygienemaßnahmen von den Betroffenen angezweifelt werden. Auch wegen diesem Aspekt ist eine Standardisierung des Vorgehens bei MRSA-Nachweis und eine entsprechende Dokumentation empfehlenswert.

Bei endemischem Auftreten von MRE kann die vorübergehende Schließung der betroffenen Station oder Abteilung notwendig werden. MRE-Patienten sollten zum frühestmöglichen Zeitpunkt aus der stationären Behandlung entlassen werden, auch bei weiterbestehender MRE-Kolonisierung. Allerdings müssen Angehörige bzw. Mitarbeiter in einer weiterversorgenden Institution frühzeitig über die MRE-Kolonisierung informiert werden. Parallel dazu sollte grundsätzlich der behandelnde Hausarzt informiert werden.

\section{MRSA-kolonisiertes Personal}

Eine prophylaktische, routinemäßige mikrobiologische Untersuchung aller Mitarbeiter auf MRE ist in der Regel nicht notwendig. Krankenhauspersonal ist im Vergleich zur Normalbevölkerung zwar häufiger nasal mit MRSA kolonisiert [33], auch bei größeren MRSA-Ausbrüchen scheint die nasale MRSA-Besiedelung des Personals aber eine untergeordnete Rolle zu spielen [33].

Bei gehäuften MRSA-Übertragungen innerhalb eines Krankenhauses sind Screening-Untersuchungen des Personals empfehlenswert.

Dennoch werden bei einem Verdacht auf MRSA-Übertragung und bei MRSA-Epidemien zur Unterbrechung möglicherweise vorhandener Infektketten Scree- ninguntersuchungen des Personals empfohlen $[7,33]$. Untersucht werden sollten dann Nasen- und Rachenabstrich. Bei mehr als zwei zeitlichen Infektfällen mit MRE sind diese Untersuchungen bei allen Mitarbeitern des betroffenen Bereiches durchzuführen, wenn ein epidemiologischer Zusammenhang evident ist oder vermutet wird.

Die Dekolonisation von betroffenem Personal erfolgt wie bei Patienten. Es sollte eine Umsetzung an einen Arbeitsplatz ohne Patientenkontakt oder eine Beurlaubung über den Zeitraum der Dekolonisation erfolgen $[7,20]$. Kritiker des Personalscreenings warnen vor einer Stigmatisierung und Störung des routinemäßigen Arbeitsprozesses [20].

Therapie der MRSA-Infektion des muskuloskelettalen Systems

Eine effektive Therapie der Wundinfektion, Protheseninfektion sowie chronischen Knochen- und Weichteilinfektion mit MRSA-Nachweis ist möglich. Sie erfordert ein konsequentes Hygienemanagement mit Isolation der Patienten und ein konsequentes chirurgisches Vorgehen.

Die grundsätzlichen chirurgischen Prinzipien und Maßnahmen bei MRSA-Infektionen unterscheiden sich dabei nicht von denen der septischen Chirurgie bei Infektionen mit „Normalkeimen“.

Eine vollständige Eradikation des MRSA ist möglich. Nach Phasen der Infektfreiheit oder Infektruhe ist jedoch jederzeit, speziell bei chronischer Osteitis, ein Infektrezidiv möglich. Die wesentlichen Faktoren, die ein Infektrezidiv hervorrufen, sind bisher nicht identifiziert. Bei Patienten mit Infektrezidiven nach MRSAInfektionen lässt sich häufig erneut MRSA nachweisen [29]. Insofern ist bei Patienten mit Infektrezidiven und MRSA-Vorgeschichte ein entsprechendes Screening vor Aufnahme oder die primäre Isolation zu empfehlen.

\section{Wundmanagement}

Das chirurgische Wundmanagement bei MRSA-Infektionen stimmt grundsätzlich mit dem Vorgehen bei Infektionen mit sensiblen Keimen überein. In der akuten putriden Phase sollten operative Revisionen in 3- bis 4-tägigen Abständen erfolgen. Bewährt hat sich die Vakuumversiegelung der Wunden. Sie verhindert den direkten Kontakt mit dem Wundsekret, 


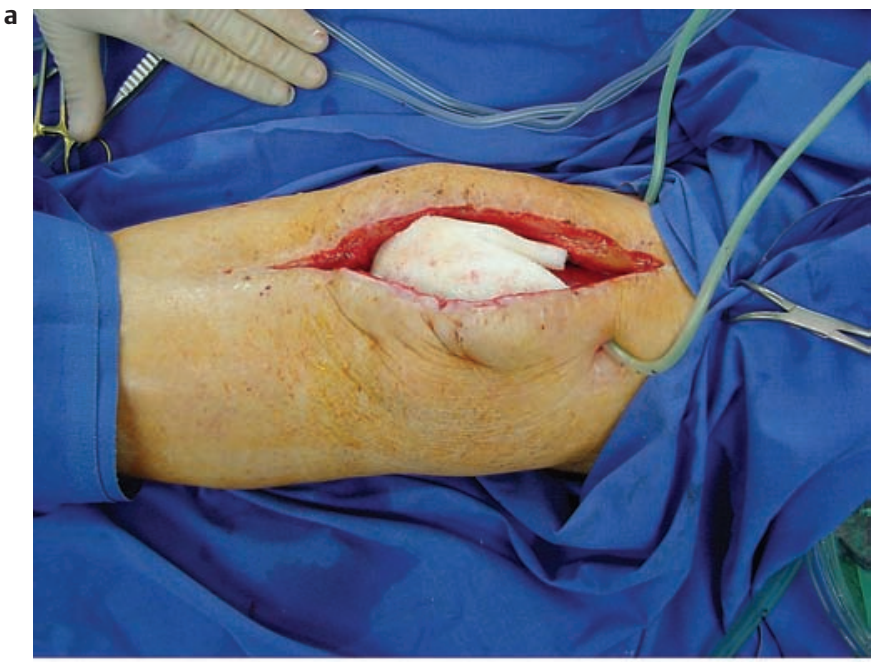

b

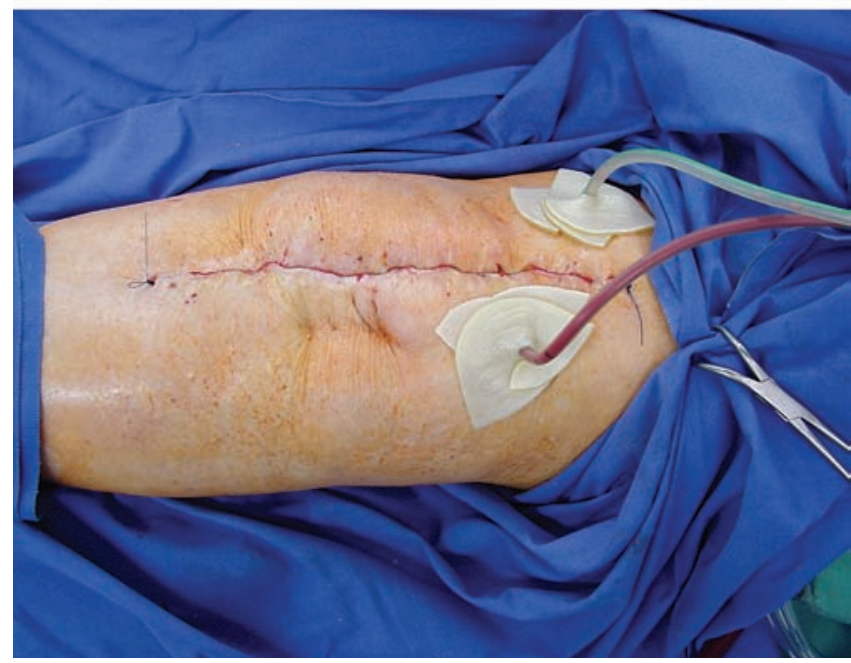

Abb.5a, b Temporärer Wundverschluss mit Vakumverbänden im Rahmen des EtappenDebridements bei Kniegelenk-TEP-Infektion und Prothesenausbau. wie er bei einer offenen Wundbehandlung auftreten könnte (Abb.5) [44]. Gleichzeitig wird mit dem Vakuumverband das Risiko einer aerogenen Übertragung vermindert sowie die unspezifische Immunantwort durch die geschlossene Wundbehandlung verstärkt $[18,26]$. Der Wundverschluss mit Polyurethanfolien vermindert zusätzlich das Bakterienwachstum von Staphylococcus aureus [27].

In unserem Vorgehen hat es sich bewährt, entsprechend der Empfehlungen der Hygiene, den definitiven Wundverschluss nach drei aufeinander folgenden, mikrobiologischen Untersuchungsergebnissen ohne MRSA-Nachweis durchzuführen. Liegt gleichzeitig keine Kolonisation von anderen Prädilektionsstellen mit MRSA mehr vor, kann die Isolation aufgehoben werden.

\section{Lokale Antibiotikatherapie}

Ergänzend können topische Antibiotika angewendet werden. Im Rahmen des Revisionsprogramms hat sich im eigenen Vorgehen der Einsatz von testgerechten, hoch dosierten kurz wirksamen Antibiotikaträgern auf Kollagenbasis bewährt. Grundsätzlich muss hierbei berücksichtigt werden, dass nahezu alle lokalen Antiseptika einen hemmenden Einfluss auf die Zellproliferation und somit auf die Wundheilung haben [38]. Limitierend wirkt, dass es erst wenig kommerziell verfügbare Medikamententräger gibt, die mit dem entsprechenden Antibiotikum dotiert sind, gegen die keine Multiresistenzen vorliegen.

\section{Systemische Antibiotikatherapie}

Weit verbreitete, häufige, ineffektive Antibiotikatherapien bedingen einen weiteren Selektionsvorteil für multiresistente Keime.
Ineffektiver Antibiotikaeinsatz führt zu einem Selektionsvorteil für multiresistente Keime.

Klinisch manifeste MRSA-Infektionen erfordern eine systemische antibiotische Therapie $[7,36]$. Hierzu stehen nur wenige Substanzgruppen zur Verfügung: die Glykopeptide Vancomycin und Teicoplanin evtl. in Kombination mit Fosfomycin oder Rifampicin sowie Qinupristin/Dalfopristin und Linezolid. Häufig sind andere Antibiotika, welche bei der Resistenztestung als empfindlich eingestuft wurden, therapeutisch unwirksam. Das erst seit kurzem auf dem Markt befindlichen Oxazolidinon Linezolid, welches derzeit noch eine sehr gute Wirksamkeit gegenüber ORSA und ORSE aufweist, muss im Hinblick auf eine Resistenzentwicklung intensiv beobachtet werden.

\section{Neue Substanzen}

Hoffnungsvolle Ansätze gibt es in der Immuntherapie der MRSA-Infektionen. Gegen die Exotoxine, Exoenzyme, Zellwandkomponenten (Peptidoglykan, Teichonsäuren) und Kapselbstandteile des Staph. aureus gibt es eine komplexe humorale Antwort. Erste klinische Studien zeigen, dass man sich diese Immunantwort therapeutisch durch einen spezifischen Antikörper zunutze machen kann [6].

\section{Ökonomische Auswirkungen}

Verschiedene Studien belegen, dass Infektionen durch MRSA-Stämme zu einer längeren Verweildauer im Krankenhaus führen und höhere direkte Kosten für Diagnostik und Therapie verursachen $[3,4,11,16]$. Die finanzielle Mehraufwendung pro MRSA-Patient auf einer Intensivstation belaufen sich auf 1622 pro Tag [11]. Die anfallenden täglichen Mehrkosten auf einer orthopädischen Normalstation wurden von Kreutzer et al. auf 536,49 eingeschätzt [25]. Die Kosten für die Sperrung von Betten für die notwendigen Isolierungsmaßnahmen an einem Großklinikum betragen entsprechend Angaben von Popp et al. 210000 pro Jahr [35]. Es wurde mit Einführung des G-DRG-Systems zum 1. Januar 2004 versäumt, die ökonomischen Rahmenbedingungen für ein effizientes MRSA-Management zu schaffen.

Die Infektionen und Kolonisationen durch MRSA werden nur unzureichend im G-DRG abgebildet. 


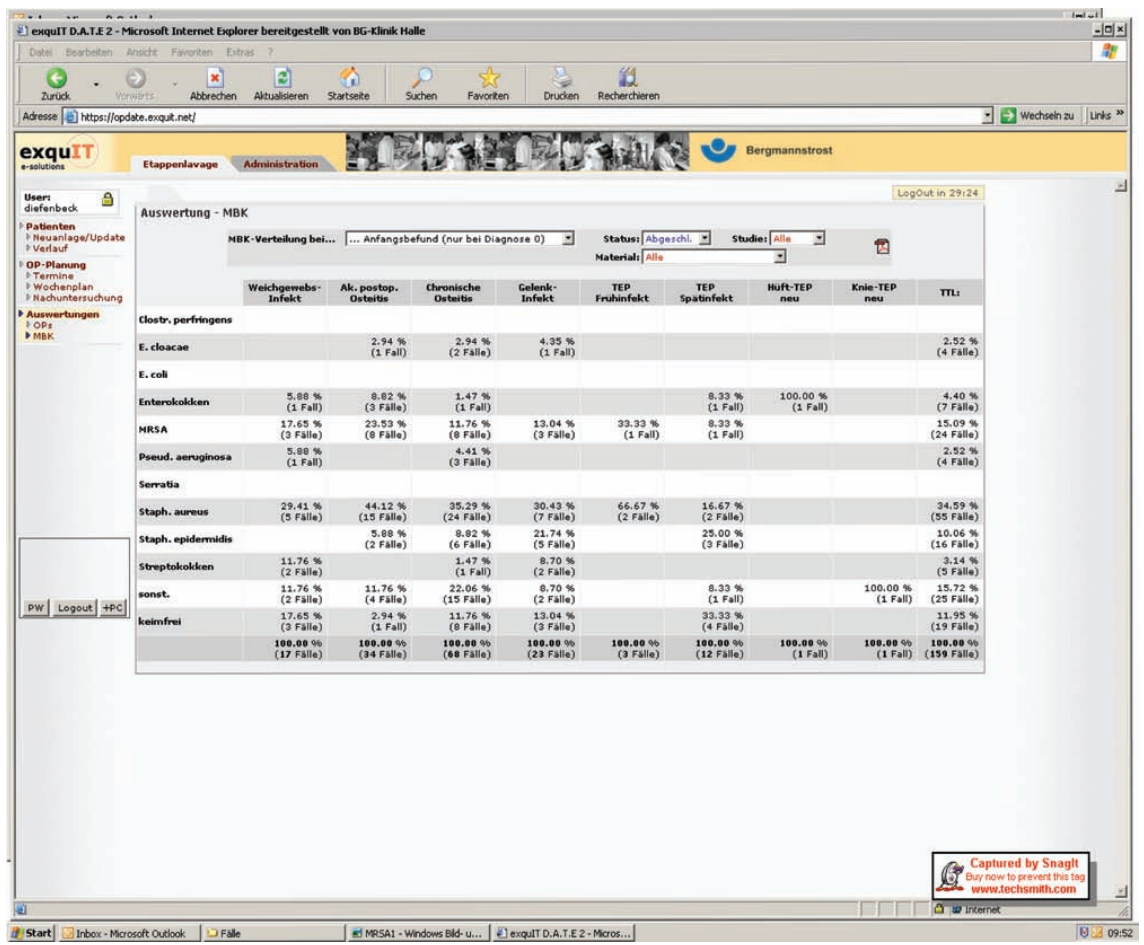

Abb. 6 Beispiel einer Internet-basierten Datenbank zur Erfassung des Keimspektrums inkl. der multiresistenten Keime bei muskuloskelettalen Infektionen.

\section{Prävention}

Der wichtigste Übertragungsweg von MRSA ist der direkte Kontakt mit den Händen [7]. Verbesserte Händedesinfektionsmaßnahmen in einem Klinikum können die MRSA-Häufigkeit reduzieren [34]. Wichtig ist jedoch auch die Kontaktisolierung von MRSA-infizierten oder -besiedelten Patienten. Staphylokokken besiedeln besonders häufig immunsupprimierte Patienten sowie Intensivpatienten. Ungefähr 70\% aller MRSA-Isolate stammen von Intensivpatienten [7]. Daher sind Hygienemaßnahmen und präventive Isolationsmaßnahmen bei MRSA-Verdachtsfällen besonders strikt auf Intensivstationen einzuhalten.

Auf unseren Intensivstationen werden zuverlegte bzw. aufgenommene Patienten mit Risikomerkmalen zunächst isoliert und bezüglich MRSA gescreent. Sind die mikrobiologischen Untersuchungen der Prädilektionsstellen unauffällig, wird die Isolation aufgehoben. Wir empfehlen außerdem das Screening ehemals MRSA-positiver Patienten. Insbesondere Patienten mit Osteitisrezidiven und MRSA-Nachweis in der Vorgeschichte sind mit hoher Wahrscheinlichkeit erneut MRSA-positiv.

Es empfiehlt sich daher das Führen einer Datei der MRSA-positiven Patienten, um bei Neuaufnahmen oder Wiedervorstellungen entsprechende Maßnahmen ergreifen zu können (Abb. 6 ). Nur mit aktiv durchgeführten Surveillance-Maßnahmen kann die Prävalenz von MRSA gesenkt werden. Dies konnte überzeugend in Dänemark demonstriert werden, wo die Rate an nosokomialen MRSA-Infektionen von $34 \%$ auf weniger als $1 \%$ gesenkt und nunmehr über mehr als zwei Jahrzehnte beibehalten werden konnte [9].

\section{Literatur}

${ }^{1}$ Anonymus. Empfehlungen zur Prävention und Kontrolle von Methicillin-resistenten Staphylococcus aureus-Stämmen (MRSA) in Krankenhäusern und anderen medizinischen Einrichtungen. Bundesgesundheitsbl Gesundheitsforsch Gesundheitsschutz 1999; 42: $954-958$

${ }^{2}$ Arens S. Osteitis - immer noch ein Problem? Trauma Berufskrankh 2002; 4: 295-305

${ }^{3}$ Back NA, Linnemann CC, Jr., Staneck JL et al.. Control of methicillin-resistant Staphylococcus aureus in a neonatal intensive-care unit: use of intensive microbiologic surveillance and mupirocin. Infect Control Hosp Epidemiol. 1996; 17: $227-231$

${ }^{4}$ Boyce JM. Should we vigorously try to contain and control methicillin-resistant Staphylococcus aureus? Infect Control Hosp Epidemiol 1991; 12: 46-54

${ }^{5}$ Bühren V. Hygienemaßnahmen in der Chirurgie. Chirurg 2002; 73: 954

${ }^{6}$ Burnie JP, Matthews RC, Carter T et al.. Identification of an Immunodominant $\mathrm{ABC}$ Transporter in Methicillin-Resistant Staphy- lococcus aureus Infections. Infect Immun 2000; 68: 3200-3209

${ }^{7}$ Daschner F, Schumpelick V. MRSA als Herausforderung an die klinische Organisation. Chirurg 2002; 73: 924-929

${ }^{8}$ Diskussion zu dem Beitrag von Kipp F. et al. „Bedrohliche Zunahme Methicillin-resistenter Staphylococcus-aureus-Stämme“. Dtsch Arztebl 2005; 102: 898-902

${ }^{9}$ Farr BM, Salgado CD, Karchmer TB et al.. Can antibiotic-resistant nosocomial infections be controlled? Lancet Infect Dis 2001; 1: $38-45$

${ }^{10}$ Fridkin SK, Hageman JC, Morrison M et al. Methicillin-resistant Staphylococcus aureus disease in three communities. N Engl J Med 2005; 352: 1436-144

${ }^{11}$ Geldner G, Ruoff M, Hoffmann HJ et al.. [Cost analysis concerning MRSA-infection in ICU]. Anasthesiol Intensivmed Notfallmed Schmerzther 1999; 34: 409-413

${ }^{12}$ Gottlieb S. CDC reports first case of vancomycin resistant Staphylococcus aureus. $\mathrm{Br}$ Med J 2003; 326: 783

${ }^{13}$ Haley RW. Methicillin-resistant Staphylococcus aureus: do we just have to live with it? Ann Intern Med 1991; 114: 162 - 164

${ }^{14}$ Hayes PD, Nasim A, London NJ et al.. In situ replacement of infected aortic grafts with rifampicin-bonded prostheses: the Leicester experience (1992 to 1998). J Vasc Surg 1999; 30: $92-98$

${ }^{15}$ Hermann M. MIQ Expertengremium Mikrobiologische-Infektiologische Qualitätsstandards: Infektionen des Knochens. 11. klinisch-mikrobiologisch-infektiologisches Symposium Berlin, Dezember 2003

${ }^{16}$ Herr CE, Heckrodt TH, Hofmann FA et al.. Additional costs for preventing the spread of methicillin-resistant Staphylococcus aureus and a strategy for reducing these costs on a surgical ward. Infect Control Hosp Epidemiol 2003; 24: 673-678

${ }^{17}$ Hornberg C, Koller A, Bühring W et al.. Methicillin-resistenter Styphylococcus aureus (MRSA). Aktuelle Situation und Bedeutung der Infektionsprävention in der Technischen Orthopädie. Orthopäde 2001; 30: 231 - 235

${ }^{18}$ Hutchinson JJ, Lawrence JC. Wound infection under occlusive dressings. J Hosp Infect 1991; 17: 83-94

${ }^{19}$ Infektionsschutzgesetz ?IfSG. Gesetz zur Verhütung und Bekämpfung von Infektionskrankheiten beim Menschen. Bundesgesetzblatt 2000; 33: 1045-1071

${ }^{20}$ Kaminski A, Rohr U, Schlösser S et al.. MRSAkolonisiertes medizinisches Personal: Opfer oder Täter? Trauma Berufskrankh 2002; 4: $350-353$

${ }^{21}$ Kilgus DJ, Howe DJ, Strang A. Results of periprosthetic hip and knee infections caused by resistant bacteria. Clin Orthop 2002; 404: $116-124$

${ }^{22}$ Kipp F, Friedrich AW, Becker K et al. Bedrohliche Zunahme Methicillin-resistenter Staphylococcus-aureus-Stämme. Dtsch Arztebl 2004; 101: 2045-2050

${ }^{23}$ Kreiswirth B, Kornblum J, Arbeit RD et al.. Evidence for a clonal origin of methicillin resistence in Staphylococcus aureus. Science 1993; 259: 227-230

${ }^{24}$ Kresken M, Hafner D, Witte W et al.. Resistenzentwicklung bei Staphylokokken und anderen grampositiven Erregern gegenüber Chemotherapeutika im mitteleuropäischen Raum. Chemotherapie Journal 2000; 9: 5 14

${ }^{25}$ Kreutzer J, Nußbaum B, Bernd L et al.. Reale Kosten der MRSA-Osteitis - eine retrospek- 
tive Studie im Zeitraum von 1996-2001. Trauma Berufskrankh 2002; 4: 344-349

${ }^{26}$ Lawrence JC: Reducing the spread of bacteria. J Wound Care 1993; 2: 48-52

${ }^{27}$ Mertz PM, Ovington LG: Wound healing microbiology. Dermatol Clin 1993; 11: $739-$ 747

${ }^{28}$ Methicillin-resistente Staphylococcus aureus (MRSA) in deutschen Alten- und Pflegeheimen - zur Situation. Epidemiologisches Bulletin RKI 2003; 19: 145-148

${ }^{29}$ Mückley T, Kirschner M, Hofmann GO et al. Management der MRSA-Wundinfektion. Hefte Unfallchir 2001; 283: 417-418

${ }^{30}$ Mückley T, Hierholzer C, Diefenbeck M et al.. Die MRSA-Infektion in der septischen Extremitätenchirurgie. Chirurg 2004; 75: 269 275

${ }^{31}$ Murphy GJ, Pararajasingam R, Nasim A et al.. Methicillin-resistant Staphylococcus aureus infection in vascular surgical patients. Ann R Coll Surg Engl 2001; 83: 158-163

${ }^{32}$ Nichols RL: Postoperative infections in the age of drug-resistant gram-positive bacteria. Am J Med 1998; 104: 11 - 16

${ }^{33}$ Peltroche-Llacsahuanga H, Haase G, Luttikken R. Methicillin-resistente Staphylococcus aureus (MRSA) - Klinische Implikationen. Chirurg. 1998; 69: 801-805

${ }^{34}$ Pittet D, Hugonnet S, Harbarth S et al.. Effectiveness of a hospital-wide programme to improve compliance with hand hygiene. Infection control programme. Lancet 2000; 356: $1307-1312$

${ }^{35}$ Popp W, Hilgenhöner M, Leisebein T et al. Personalkosten durch Isolierungsmaßnah- men von MRSA-Patienten. Gesundheitsökonomie und Qualitätsmanagement 2003; 8: $187-190$

${ }^{36}$ Stark M, Heeg P. Klinische Aspekte von Infektionen durch Methicillin-resistente Staphylococcus aureus. Akt Traumatol 2002; 32: $258-260$

${ }^{37}$ Stille W, Simon C. Staphylokokken-Infektionen. In: Stille W, Simon C (Hrsg.): Antibiotika-Therapie in Klinik und Praxis. Schattauer, Stuttgart, 2000: 377-380

${ }^{38}$ Teepe R, Koebrugge E, Löwik C et al.. Cytotoxic effects of topical antimicrobial and antiseptic agents on human keratinocytes in vitro. J Trauma 1993; 35: 8-19

${ }^{39}$ Tenover FC, Arbeit RD, Goering RV. How to Select and Interpret Molecular Strain Typing Methods for Epidemiological Studies of Bacterial Infections: A Review for Healthcare Epidemiologists. Infect Control Hosp Epidemiol 1997; 18: 426-439

${ }^{40}$ Tsiodras S, Gold HS, Sakoulas G et al.. Linezolid resistance in a clinical isolate of Staphylococcus aureus. Lancet 2001; 358: 207-208

${ }^{41}$ Wichelhaus TA, Schäfer V, Brade V. Typisierungsverfahren in der Infektionsepidemiologie. Chemotherapie Journal 2000; 9: 93-98

${ }^{42}$ Witte W, Braulke C, Heuck D. MRSA-Situation in Deutschland. Hyg Med 2000; 25: 347 350

${ }^{43}$ Witte W: Epidemiologisches Bulletin, Robert Koch-Institut 35/2003

${ }^{44}$ Young T: Methicillin-resistant Staphylococcus aureus. J Wound Care 1996; 5: 475-477

\section{Dr. med. Thomas Mückley}

Oberarzt

Dr. med. Michael Diefenbeck

Assistenzarzt

Prof. Dr. med. Dr. rer. nat.

Gunther 0. Hofmann

Direktor

Friedrich-Schiller-Universität Jena

Klinik für Unfall-, Hand- und Wiederherstellungschirurgie

Erlanger Allee 101

D-07747 Jena 